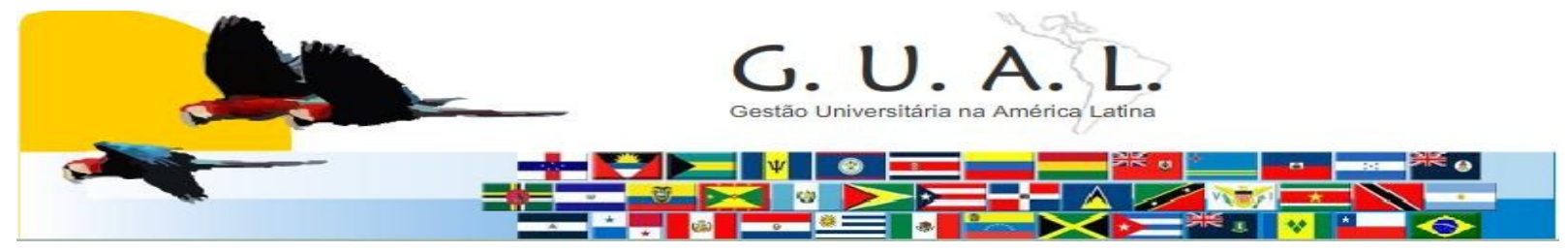

ISSN 1983-4535

\title{
GESTIÓN ACADÉMICA Y ESTRATEGIAS PARA LA RETENCIÓN DE LA MATRÍCULA: PROGRAMAS DE TUTORÍAS EN LA UNIVERSIDAD PÚBLICA
}

\author{
Marta Arana, UNMDP \\ Karina Bianculli, UNMDP \\ Ernesto Uriondo, UNMDO
}

\begin{abstract}
RESÚMEN
El siglo XXI enfrenta a la Gestión en la universidad pública con nuevos desafíos. En el área académica, una problemática insoslayable es el tema del abandono de los alumnos, muy destacada en los primeros años de las carreras que cursan. La problemática del abandono de los alumnos en la universidad pública argentina es un tema prioritario en la Educación Superior. En la actualidad se nos presenta una población estudiantil crecientemente heterogénea en su formación sociocultural y en su composición sociodemográfica (sexo, edad), siendo un desafío avanzar hacia la inclusión, manteniendo la calidad educativa en un contexto socioeconómico de creciente desigualdad, donde los estudios superiores desaparecen del horizonte de expectativas de miles de jóvenes año a año. Desde la década de los 80 del siglo pasado se percibe un gran interés por parte de las autoridades académicas por crear Servicios y Programas de Orientación Universitaria, que van más allá de la mera información y con lo que se busca una mejor preparación y un desarrollo más integral de los jóvenes para la transición a la vida social activa. Desde el año 2006 el Grupo de investigación de la UNMDP, Programa Universidad analiza el desgranamiento de la matrícula universitaria. A través de estos años se ha realizado una profunda investigación a través de la metodología cuali-cuantitativa sobre el universo de alumnos. Pudiéndose definir su perfil y las dificultades más habituales como el acceso de los aspirantes que provienen de la educación media pública, la lentificaciòn en la carrera y el abandono, principalmente en el primer año. La presente ponencia tiene como objetivo principal presentar ante la comunidad académica las experiencias en torno a la implementación del Programa Piloto de Tutores Iniciales que viene desarrollándose en el ámbito de la Facultad de Ciencias Económicas y Sociales, de la UNMDP
\end{abstract}

Palabras clave: desgranamiento, matrícula universitaria, tutorías. 


\section{GESTIÓN ACADÉMICA Y ESTRATEGIAS PARA LA RETENCIÓN DE LA MATRÍCULA: PROGRAMAS DE TUTORÍAS EN LA UNIVERSIDAD PÚBLICA}

\section{INTRODUCCIÓN}

El siglo XXI enfrenta a la Gestión en la universidad pública con nuevos desafíos. En el área académica, una problemática insoslayable es el tema del abandono de los alumnos, muy destacada en los primeros años de las carreras que cursan.

A partir de los años noventa, la Universidad Publica Argentina, fue protagonista de un largo debate que se extendió durante esta década. La calidad educativa y la descentralización, fueron dos temas recurrentes en los congresos y reuniones del área. Sin embargo, dejando de lado, la construcción de esta agenda universitaria, que se relaciona con la planta docente y el nivel institucional, que posibilitaría pensar a la Universidad del siglo XXI, un problema como el abandono y la lentificación de sus estudiantes empezó a surgir entre los congresos y finalmente en la construcción de la nueva agenda .

En un contexto de amplia desigualdad social y reiteradas crisis económicas, que año a año expulsan a cada vez más personas al abismo de la pobreza estructural, que lleva varias generaciones en algunos casos. Aspectos que no se pueden dejar de lado para pensar las posibilidades reales de ingreso y permanencia de los alumnos que forman parte de las actuales cohortes. Sin embargo los adolescentes que nutren las aulas de primer año, no son individuos que provienen de grupos en emergencia social y económico, sino son hijos de una clase media empobrecida, de padres profesionales y/o empleados calificados, que construyeron las esperanzas de movilidad y estabilidad socioeconómica en la formación de sus hijos. Padres que pagaron una educación, tal vez mediocre, pero disciplinada para sus hijos, que hoy acceden a la Universidad Publica a cursar largas carreras con, en la mayoría de los casos, la inclinación a las profesiones liberales.

Cabe preguntarnos sobre quienes acceden a la Universidad hoy, y están dispuestos a transitar el trayecto educativo, que implica largos años de su vida en una actividad que no permite, en la mayoría de los casos, otra ocupación posible, además de saber con anticipación que no asegura un lugar en el conflictivo y cambiante escenario del mercado laboral. Qué lugar simbólico, ocupa la formación superior en el horizonte de expectativas de las metas personales imaginadas de estos alumnos, sus familias y su generación de amigos y compañeros.

Una antigua actividad docente, como la tutoría surge, renovada como parte de las medidas que se piensan desde la institución para retener su alumnado y posibilitar que a un 


\section{GESTIÓN ACADÉMICA Y ESTRATEGIAS PARA LA RETENCIÓN DE LA MATRÍCULA: PROGRAMAS DE TUTORÍAS EN LA UNIVERSIDAD PÚBLICA}

amplio número de alumnos que comienzan una carrera universitaria, les sea posible finalizarla en tiempo y forma.

\section{Antecedentes históricos I: La Universidad}

Para acercarnos a la universidad como marco / actor, es imprescindible un análisis bibliográfico acerca de las diferentes visiones que se tienen de ella. Entre las más importantes se puede citar la que sostiene que el espíritu de las Universidades es la universalización del conocimiento, como propone Burton Clark. ${ }^{1}$

Pero no se puede negar que, para una institución creada hace cerca de mil años, para la consagración y legitimación de conocimientos y saberes, tanto su rol iniciático como su articulación con la Sociedad, han ido cambiando al igual que su derrotero histórico.

En sus orígenes, la universidad medieval buscaba, en coherencia con su tiempo histórico, la transmisión del conocimiento o saber absoluto, el respeto a la verdad y el desarrollo del espíritu de estudio. Como arquetipo de universidad docente, el papel del profesor era guiar, orientar, tutorizar la conducta moral, social e intelectual de sus alumnos en la búsqueda del conocimiento, como vía para el crecimiento personal, en un marco de relación personal estrecho.

Sin perder el valor de la búsqueda de la verdad científica, el saber por el saber, la universidad renacentista aunará enseñanza e investigación entre sus fines. El espíritu científico y de libertad de los docentes inunda las universidades, donde lo importante será la reflexión crítica de los conocimientos adquiridos. Si bien, la concepción estrictamente docente de los centros universitarios será históricamente difícil de superar hasta el siglo XIX.

Es posible delimitar la íntima relación de la organización "universidad" con la constitución de los Estados nacionales, la secularización de la sociedad y la expansión de la Revolución Industrial a comienzos del siglo XIX, cuando se comenzó a demandar a esta Institución la generación de saberes técnicos para su incorporación al sector industrial. A su vez se convirtió en difusora de ideas y valores de la cultura moderna. Como resultado de estos procesos se advierte la nacionalización de las universidades, y en ella la constitución de modelos universitarios. Entre estos últimos, se pueden diferenciar: a) la universidad humboltiana con el principio rector de la investigación y la ciencia; b) la universidad

\footnotetext{
1 ...En principio los centros de investigación y educación superior comparten las ideas de universalización del conocimiento. La búsqueda desinteresada de la verdad ( o lo que podríamos denominar, en un lenguaje más epistemológico, la formulación permanente de nuevas hipótesis sobre la realidad objetiva) se encuentra en el corazón de la vida académica... (Burton Clark)
}

Rev. GUAL., Florianópolis, v.2, n. 1, p.16-34, ano 2009. 


\section{GESTIÓN ACADÉMICA Y ESTRATEGIAS PARA LA RETENCIÓN DE LA MATRÍCULA: PROGRAMAS DE TUTORÍAS EN LA UNIVERSIDAD PÚBLICA}

napoleónica con la finalidad de la formación de profesionales para la burocracia estatal recientemente creada y c) la universidad norteamericana abierta a las demandas del mercado y la investigación. A través de esta breve síntesis se observa la estrecha relación entre la matriz sociopolítica y la universidad.

La UNMDP es una universidad relativamente joven, es posible rastrear su incipiente presencia en diciembre de 1955 cuando se conformó una junta Pro-Universidad del Mar que solicitó al Ministro de Gobierno de la provincia de Buenos Aires el establecimiento de facultades en la ciudad. Las aspiraciones eran crear: la Facultad de Arquitectura e Ingeniería Naval, la Facultad de Ciencias Económicas, el Instituto de Biología Marina, la Facultad de Medicina, el Museo Oceanográfico y Acuarium, la escuela Superior de Artes Plásticas y Decorativas, la Facultad de Derecho, el Museo de Ciencias Naturales, la Escuela Tecnológica de Pesca y la Escuela Superior de Agricultura y Frutihorticultura. En el año 1958 se creó el Instituto Universitario Libre, que fue el primer centro local de estudios superiores, dependiente del obispado. Tiempo después se transformaría en la Universidad Católica. En el año 1961 se creó por el decreto 11.723 la Universidad de la Provincia de Buenos Aires, donde se inscribieron 197 alumnos, inaugurando sus clases el 12 de enero de 1962 con la presencia del entonces presidente de la Nación Arturo Frondizi. En el año 1975 se sancionó la Ley que creó la UNMDP y desde el 1 de enero de 1976 se fusionó la Universidad Católica y la de la Provincia de Buenos Aires. ${ }^{2}$

La Facultad de Ciencias Económicas y Sociales es una facultad compuesta por cuatro carreras: Contador Público, Licenciatura en Economía, Licenciatura en Administración de Empresas y Licenciatura en Turismo. Se caracteriza por contar con una planta docente que ejerce su profesión además de la docencia.

\section{Antecedentes Històricos II: La Acción Tutorial}

La tutoría universitaria no es un invento del siglo XX. Una mirada retrospectiva a la historia de esta institución confirma que la función tutorial, entendida como el acompañamiento de los discentes en la formación de lo que podría denominarse un "estilo universitario", ha formado parte consustancial de la propia tarea docente desde los albores de

\footnotetext{
${ }^{2}$ Edición especial Centenario 100 años del Diario la Capital , 2005.
} 
la universidad. Pero su definición va ligada a las diferentes concepciones o modelos de universidad desarrollados en contextos temporales y geográficos diferenciados.

La Tutoría Universitaria se entiende como una acción de intervención formativa destinada al seguimiento académico de los estudiantes, desarrollada por profesores como una actividad docente más, si bien con el apoyo, coordinación y recursos técnicos facilitados por el profesorado especializado o personal técnico. Debe considerarse como una acción nuclear dentro del conjunto de acciones impulsadas en todas las universidades para ayudar a resolver las encrucijadas entre cantidad y calidad, entre la masificación y la personalización, entre la gestión del profesor y la gestión del alumno, entre el énfasis por el resultado y énfasis del proceso. $^{3}$

\section{La tutoría de los diferentes modelos de universidad}

La relación que existe entre los fines de la universidad y la función tutorial desarrollada por el profesorado va más allá de la división histórica. Se destacan tres modelos relacionados a los arquetipos de universidad señalados anteriormente:

\section{Modelo Académico}

Ligado a la tradición alemana centra las funciones de la universidad en el desarrollo académico de los estudiantes, sin un fin profesionalizados y en el estímulo de la ciencia.

\section{Modelos de Desarrollo Personal}

Vinculado a la tradición anglosajona la universidad atiende el desarrollo personal y el bienestar del alumno incluyendo la orientación académica, profesional y personal. Se caracteriza por la concepción de una formación más generalista y con poca relación con el mercado laboral.

\section{Modelo de Desarrollo Profesional}

Surgido del traspaso del desarrollo de la empresa o de las escuelas técnicas, el papel del profesor tutor es asegurar la capacitación profesional y el ajuste al mercado laboral

\footnotetext{
${ }^{3}$ RODRIGUEZ ESPINAR. S, 2004 Manual de tutoría universitaria. Recursos para la acción. Ed. Octaedro, Barcelona.
} 


\section{La Acción Tutorial en el siglo XXI}

Desde la década de los 80 del siglo pasado se percibe un gran interés por parte de las autoridades académicas por crear Servicios y Programas de Orientación Universitaria, que van más allá de la mera información y con lo que se busca una mejor preparación y un desarrollo más integral de los jóvenes para la transición a la vida social activa.

Desde las necesidades de orientación para acceder a la información (desarrollo de estrategias para el uso de la información) pasando por las necesidades relacionadas con la movilidad y los intercambio académicos y laborales (informar, difundir, asesorar sobre posibilidades de movilidad) las nuevas exigencias sociolaborales vigentes en la sociedad postindustrial ( para poder enfrentar de manera satisfactorias las sucesivas elecciones vocacionales), no solo se les va a demandar a los estudiantes graduados que sepan y que sepan hacer, sino que además sepan estar y sepan ser, estas últimas capacidades sólo se fomentan de orientación orientadora.

En esta perspectiva se entiende que orientar no es guiar, aconsejar, conducir, seleccionar o corregir el campo de opciones. Por el contrario, orientar es ampliar el marco de experiencias, expectativas y oportunidades. Esto permite al alumno pueda conocer una diversidad de alternativas posibles y pueda valorar la distinta fuentes de datos, antes de decantarse de forma consciente y autónoma por aquellas que considere más adecuadas y relevantes.

Por tanto, una Orientación que forme para la autonomía, para el análisis crítico de la realidad y para la adaptación a los cambios vertiginosos que suceden en la sociedad actual.

La tutoría universitaria concebida desde una visión educativa podría definirse como un proceso educativo de acciones formativas y secuenciadas, estrechamente vinculadas a la práctica educativa y con una clara proyección hacia la madurez global del individuo, mediante las cuales se enseña a aprender, comprender, reflexionar y decidir de manera comprometida, responsable y autónoma.

\section{Estado de la Cuestión y Definición del problema.}

La deserción es un problema complejo, y las causas que la provocan pueden estar originadas en una multiplicidad de motivos. Se entiende por desertar, desamparar, abandonar las obligaciones o los ideales. Se llama deserción al abandono de los estudios formales de una 


\section{GESTIÓN ACADÉMICA Y ESTRATEGIAS PARA LA RETENCIÓN DE LA MATRÍCULA: PROGRAMAS DE TUTORÍAS EN LA UNIVERSIDAD PÚBLICA}

determinada carrera. Este abandono puede ser provocado por distintos motivos: personales, familiares, económicos o sociales. Los distintos factores que afectan la deserción se pueden clasificar, en relación a la institución como exógenos o endógenos (García de Fanelli, 2002).

Datos estadísticos recientes expresan que sólo el 19\% de los estudiantes llegan a graduarse. Ese valor se mantiene casi constante desde hace una década. Estas cifras de deserción Universitaria constituyen en sí mismas un grave problema, pero no sólo para los alumnos que abandonan ${ }^{4}$ sus carreras, sino para las Universidades. El cuadro es desigual según las carreras y establecimientos superiores. Así, por ejemplo, Odontología exhibió el más alto promedio en relación ingresantes / egresados que osciló entre el 30\% y el 50\%. En cambio las Licenciaturas en Sistemas de Información, Administración y Comunicaciones fluctuaron entre el 5\% y el 13\%. Otros indicadores como la femeneización de la matrícula y una diferencia sustantiva entre las instituciones públicas y privadas son factores a considerar.

La deserción de los estudiantes universitarios en la Argentina es del 43\%. Eso afirma una investigación realizada por el Ministerio de Educación a partir de la Encuesta Permanente de Hogares (INDEC) y recién publicada por la Universidad de Tres de Febrero. "El rendimiento no es tan pobre como se creía. Está dentro de los parámetros esperables en todo el mundo, aunque hay un problema grave: la deserción está creciendo" dice José Landi, asesor de la Secretaría de Educación Superior y autor del trabajo junto con Roberto Giuliodori, investigador de la Universidad de Córdoba.

Los especialistas en educación continúan sosteniendo que las carencias de destrezas intelectuales son las responsables de la deserción en la Universidad, por lo cual los factores que influyen en el abandono de los estudios provienen del alumno y su contexto sociocultural; desatendiendo otras variables como los bajos niveles de interacción personal con profesores y otros estudiantes dentro y fuera de las aulas.

Además a la hora de la investigación en deserción estudiantil, son pocas las referencias al carácter colectivo del abandono, en el sentido de priorizar el análisis de los casos de manera aislada. Sin embargo cuando se analizan las cohortes se puede verificar características comunes en los grupos de alumnos, no solo hacia adentro del grupo observado sino también respuestas similares al entorno sociohistórico, donde determinada situación

\footnotetext{
${ }^{4}$ Abandonar, no es lo mismo que desertar que se relaciona con un discurso y un significado militar de carácter punitivo, ya que desertar se asocia a la idea de traición a la patria y el compromiso de su defensa. Marta Panaia. Directora del laboratorio MIG UTNGP. $2^{\circ}$ Encuentro de Laboratorios de Monitoreo de Inserción de Graduados.
}

Rev. GUAL., Florianópolis, v.2, n. 1, p.16-34, ano 2009. 


\section{GESTIÓN ACADÉMICA Y ESTRATEGIAS PARA LA RETENCIÓN DE LA MATRÍCULA: PROGRAMAS DE TUTORÍAS EN LA UNIVERSIDAD PÚBLICA}

económica o social uniforma respuestas. Cada alumno, no está sólo en las decisiones que toma durante el transcurso de su desarrollo educativo sino acompañado por su generación. Esta experiencia genera una identidad construida y compartida por sus integrantes.

\section{La historia de un proyecto de investigación - acción: Programa Piloto Tutores Iniciales}

En el transcurso del año 2006, el grupo de Investigación Programa Universidad (PU), que viene desarrollando sus actividades en la Facultad de Ciencias Económicas y Sociales de la UNMDP. A través de los periódicos relevamientos de la matrìcula universitaria, proyectos de becarios en la construcción de Biografías Educativas, y luego de la construcción de la herramienta metodológica, Encuesta Permanente de la Matrìcula Universitaria (EPMU),y entrevistas en profundidad, que permite la generación constante de información cuantitativa y cualitativa sobre las cohortes de alumnos. Comienza a analizar las variables que influyen sobre el abandono y la permanencia de los alumnos en esta Facultad. Entre las conclusiones prelimirares que se obtuvieron se cuentan las siguientes:

- Alto porcentaje de abandono durante los primeros años de la carreras de Contador Público, Lic. en Economía, Lic. en Administración de Empresas y Lic. en Turismo de la FCEyS.

- Alto porcentaje de procedencia de escuelas privadas de nuestros alumnos.

- Alto porcentaje de dificultad para concretar la graduación, relacionados al ritmo de estudio y evaluación.

- Alto porcentaje de lentificación.

A través de estos resultados preliminares el grupo es convocado por la gestión y uniendo esfuerzos con la Institución, el centro de estudiantes y el Grupo de Investigación PU, es puesto en marcha el Programa Piloto de Tutores Iniciales que se planteaba como objetivo central:

- Acompañar con tutorias el desarrollo de la cursada a los alumnos en los tramos iniciales de las cuatro carreras que conforman la FCEyS.

Pero se necesitaba formar los recursos del área en cuestión. Nuestra Universidad no contaba en sus antecedentes con una figura como la del tutor.

Esta práctica no era llevada a cabo de manera sistemática por ninguna de las carreras en curso. Quizá la figura que más se le parecía en el medio, era la del maestro- discípulo en 


\section{GESTIÓN ACADÉMICA Y ESTRATEGIAS PARA LA RETENCIÓN DE LA MATRÍCULA: PROGRAMAS DE TUTORÍAS EN LA UNIVERSIDAD PÚBLICA}

los finales de las carreras y en los tesistas de posgrado. La convocatoria para tutores reunió a docentes, graduados, alumnos avanzados en las carreras, integrantes del centro de estudiantes que estaban analizando estos mismos problemas, integrantes de la gestión, y todos los integrantes del grupo de Investigación PU.

En una primera instancia, durante el año 2007, se analizaron las posibilidades de ejecución del Proyecto y se realizaron charlas informativas sobre su instrumentación. Comunicar la información y la metodología de análisis, puso en agenda el tema del abandono de los alumnos.

El abandono adquirió visibilidad como problema y también en torno a su puesta en debate, surgieron las posibilidades de resolución. Ya se contaba con la experiencia que venía realizando la FRA-UTN desde hace siete años sobre el desgranamiento y la retención de la matrícula en el Programa Fénix. ${ }^{5}$

A partir de la elaboración y discusión de materiales de trabajo, donde se puso al corriente el marco teórico-práctico a los tutores se dio comienzo al programa con el comienzo de las clases en marzo de 2008.

Como primera medida se realizó una conferencia a los alumnos ingresantes a al FCEyS $^{6}$ dónde se presentó el programa y se efectuó una encuesta. La misma arrojó resultados antes vislumbrados de manera parcial. La cohorte 2008, sería la primera en observarse de manera continua y regular. Los resultados parciales de la misma son los siguientes:

\footnotetext{
${ }^{5}$ El Programa Fénix es en líneas generales un Programa de retención institucional que se comporta como sistema tutorial diversificado en su modalidad. Adopta la forma de conjuntos de proyectos específicos que actúan en diferentes niveles de las carreras de ingeniería.

También se lo puede pensar como un dispositivo de detección, acción y corrección asociado a la Secretaría Académica de la Facultad. Por otro lado es una unidad de investigación que, como área identifica, implementa y difunde estrategias y modos de intervención, en función de la permanencia y participación plena de jóvenes en el sistema educativo. ${ }^{5}$

${ }^{6}$ La población encuestada es de 405 alumnos correspondientes a las carreras de Contador Público, Lic. en Economía, Lic. en Administración de Empresas y Lic. en Turismo de la cohorte 2008
}

Rev. GUAL., Florianópolis, v.2, n. 1, p.16-34, ano 2009. 
1) Edad

\begin{tabular}{|c|c|}
\hline Edad & Cantidad de alumnos \\
\hline No contesta & 3 \\
\hline 17 & 63 \\
\hline 18 & 205 \\
\hline 19 & 57 \\
\hline 20 & 29 \\
\hline 21 & 15 \\
\hline 22 & 15 \\
\hline 23 & 5 \\
\hline 24 & 1 \\
\hline 26 & 1 \\
\hline 27 & 2 \\
\hline 28 & 2 \\
\hline 30 & 1 \\
\hline 31 & 1 \\
\hline 33 & 2 \\
\hline 37 & 1 \\
\hline 46 & 2 \\
\hline
\end{tabular}

Los resultados que arrojan la tabla resaltan que el 50,6 \% del alumnado tiene 18 años, sumados a los alumnos que poseen 17 y 19 años suman un total de 80,3\%. Esto nos informa sobre que la mayoría de los jóvenes que ingresan a la Universidad, lo hacen viniendo directamente desde la escuela media.

2) Sexo

\begin{tabular}{|c|c|c|}
\hline & Cantidad de Alumnos & Porcentaje \\
\hline No contesta & 3 & 0,74 \\
\hline Masculino & 194 & 47,90 \\
\hline Femenino & 208 & 51,36 \\
\hline
\end{tabular}

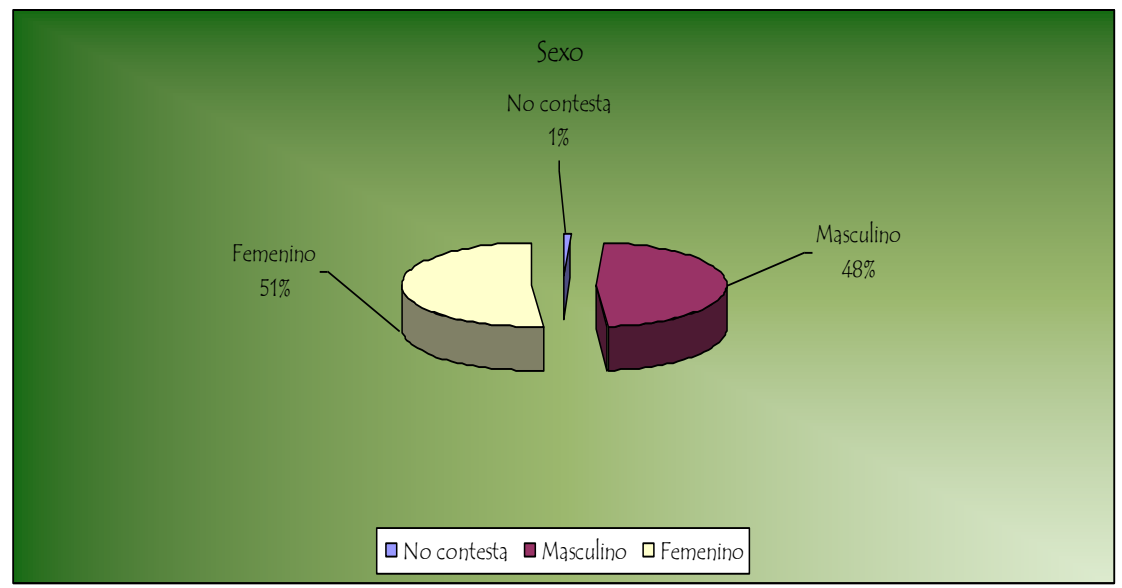

Rev. GUAL., Florianópolis, v.2, n. 1, p.16-34, ano 2009. 


\section{GESTIÓN ACADÉMICA Y ESTRATEGIAS PARA LA RETENCIÓN DE LA MATRÍCULA: PROGRAMAS DE TUTORÍAS EN LA UNIVERSIDAD PÚBLICA}

A diferencia de lo que sucede en los años avanzados de las distintas carreras ofrecidas por la FCEyS de la UNMDP, no se observan grandes diferencias de género. La femeneización de la matrícula es un fenómeno palpable en los años posteriores al ingreso.

\section{3) Tipo de Institución de origen}

\begin{tabular}{|c|c|c|}
\hline & Frecuencia & Porcentaje \\
\hline No contesta & 3 & 0,74 \\
\hline Pública & 130 & 32,10 \\
\hline Privada & 272 & 67,16 \\
\hline
\end{tabular}

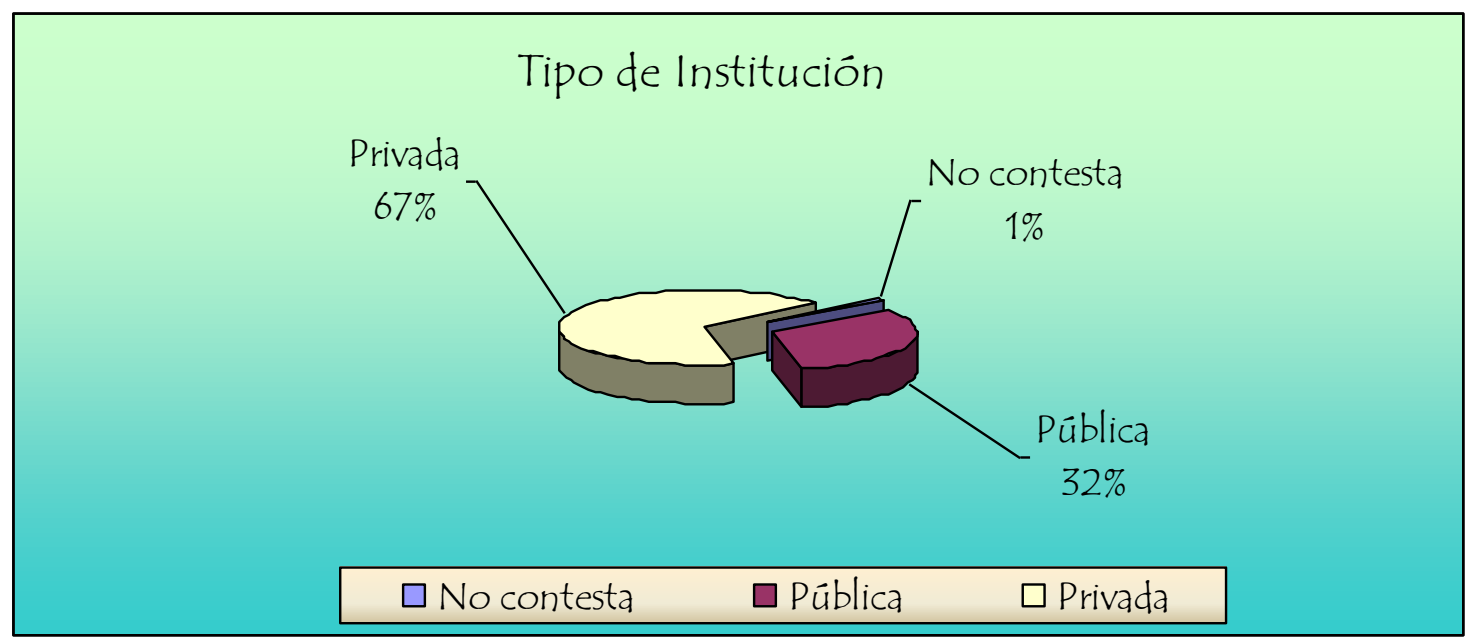

Los alumnos que han aprobado el examen de ingreso provienen, en su mayoría de colegios privados. Entre ellos se destacan el Instituto Peralta Ramos 5,19 \%, el Colegio Don Orione 4,9 \% y Fray M. Esquiu con el 3,95 \%; por otra parte, entre las escuelas públicas que proveen mayor cantidad de alumnos se encuentra en Colegio Nacional Arturo Illia con el 4,44 \%, la E.E.M. N 3 y la Escuela Carmelo Sánchez de Balcarce. Respecto a los alumnos provenientes del Colegio Nacional A. Illia, es importante destacar que ingresaron a través de un riguroso sistema de exámenes a la formación media, por lo cual, no representa una escuela pública media. De esta manera el porcentaje de escuelas públicas de origen se reduce. 


\section{4) Información sobre la carrera}

\begin{tabular}{|c|c|}
\hline & Frecuencias \\
\hline No contesta & 334 \\
\hline Desde la escuela o colegio de origen & 155 \\
\hline $\begin{array}{c}\text { Desde los medios locales de } \\
\text { información }\end{array}$ & 37 \\
\hline Amigos, compañeros o familiares & 202 \\
\hline Desde la misma Universidad & 82 \\
\hline
\end{tabular}

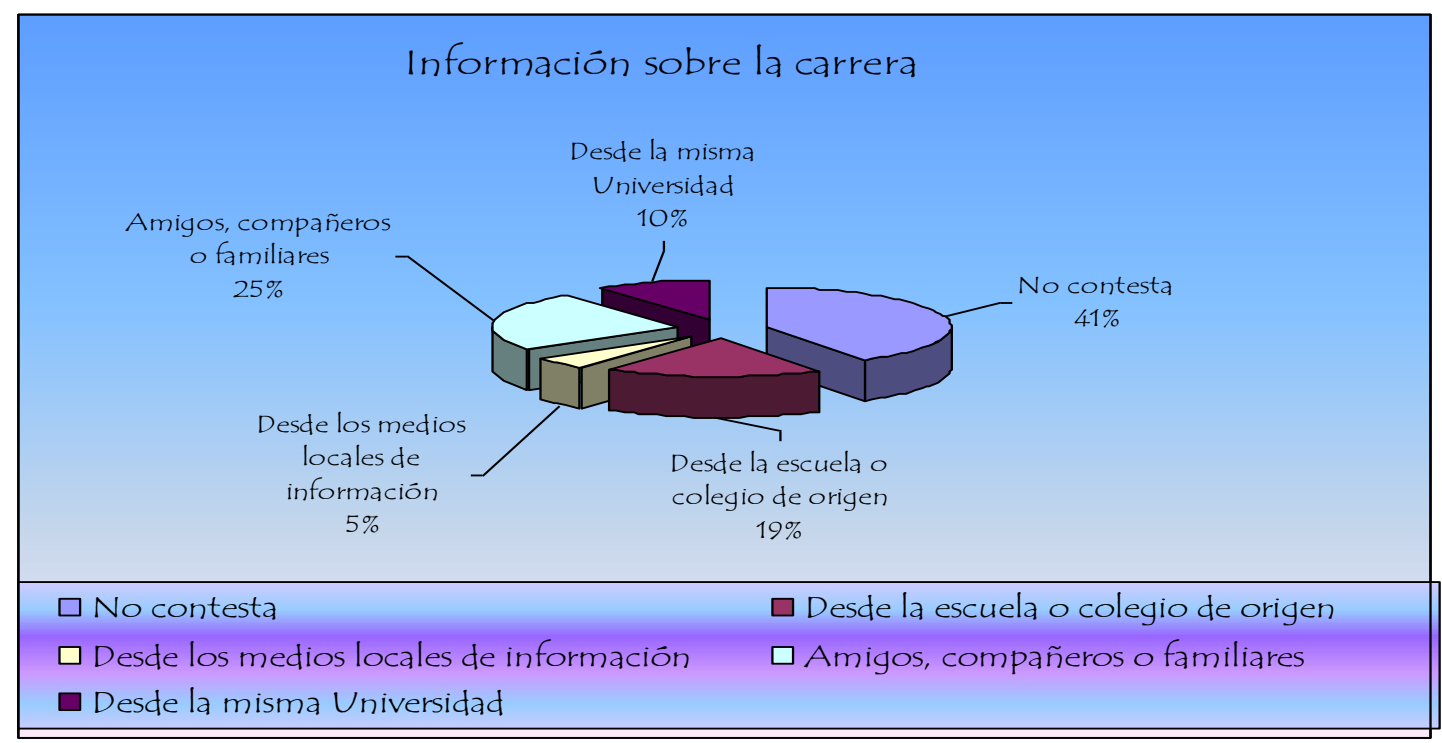

En éste punto, se tomaron como respuestas válidas las dos primeras elecciones hechas por el encuestado. Si se suman las frecuencias, éstas duplican al total de las encuestas y de la suma se desprenden los porcentajes expresados en el gráfico. Una de las observaciones hechas por los encuestados que no están presentes en dicho informe es la alusión a la Muestra Educativa realizada en el teatro Auditórium.

En este punto de la encuesta en particular, se observa que la mayoría de los encuestados obtiene información del ingreso en la Universidad desde la escuela de origen y desde el ámbito familiar. Información que no llega a los aspirantes desde los medios de comunicación o la publicidad de la propia Universidad. En este sentido, es importante reflexionar sobre el valor simbólico conferido a la formación superior en el horizonte de expectativas de los encuestados, donde en el contexto sociocultural donde se desarrollan, la formación superior es un camino posible y esperable de su desarrollo personal. 


\section{5) Elección de la carrera}

\begin{tabular}{|c|c|}
\hline Meta personal & 220 \\
\hline Decisión de mucho tiempo atrás & 177 \\
\hline Salida laboral & 124 \\
\hline Interés o vocación por temas afines & 55 \\
\hline Influencia de los padres & 22 \\
\hline Tradición familiar & 17 \\
\hline
\end{tabular}

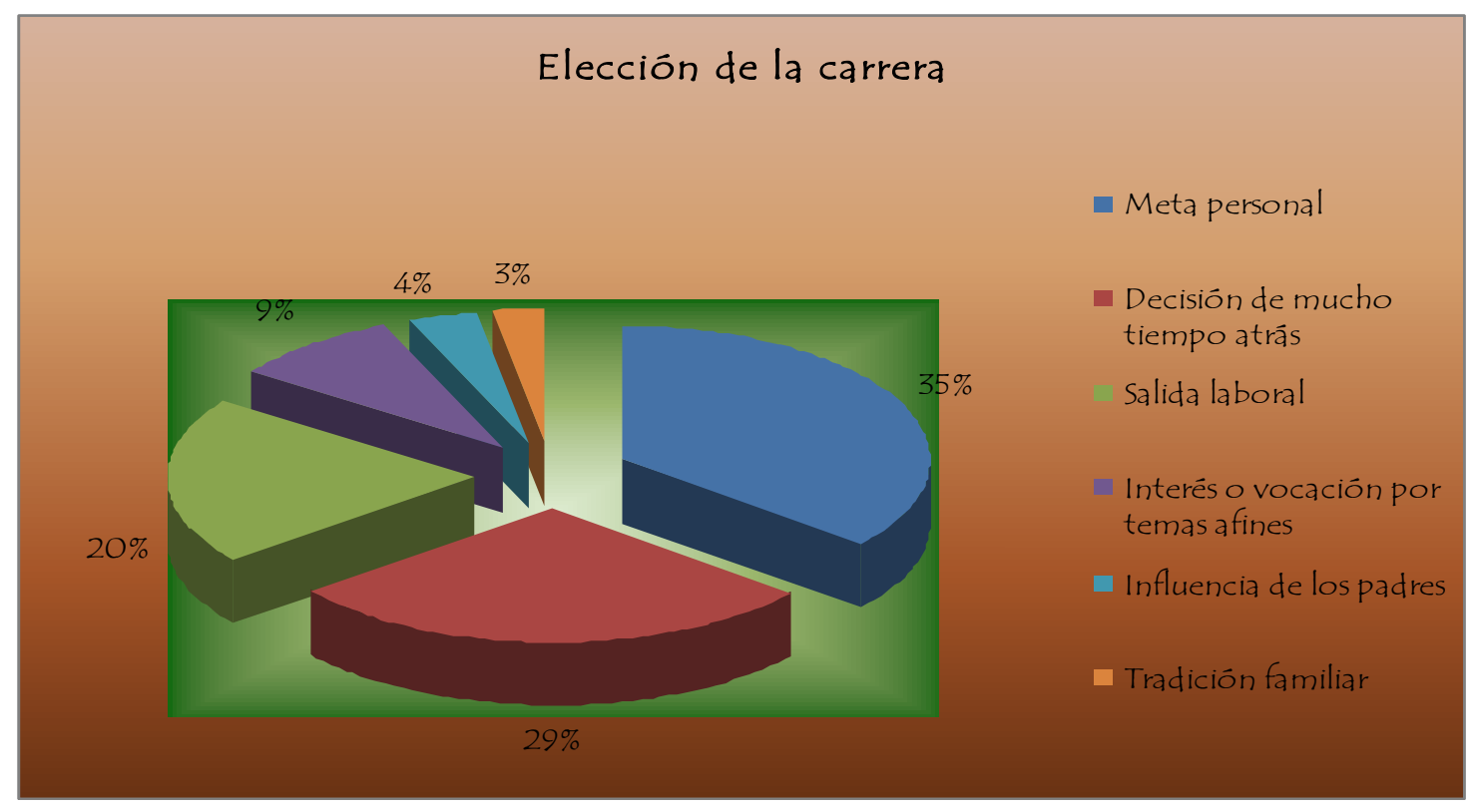

La información volcada en el gráfico resume la elección de las tres primeras variables de cada encuesta. A los datos obtenidos se le han restado el porcentaje no contestado, dando cómo resultado las frecuencias que indica la tabla.

En cuanto al punto 5 de la encuesta se relaciona directamente con el punto anterior. Las tres respuestas más concurridas, nos ayudan a entender las motivaciones y la trama de significados que sostienen la decisición de los jóvenes a estudiar una carrera universitaria. La respuesta más elegida fue la meta personal. Habría que preguntarse, qué significa meta personal para estos adolescentes. Si un desafío para el ingreso a la vida adulta, para asegurarse un lugar en el cada vez menor espacio para el ascenso social, o una preparación para el mercado laboral que exige una amplia y flexible capacitación, como responde la opción de salida laboral, sin embargo si se relacionan estas respuestas a la tercera y también muy elegida decisión de mucho tiempo atrás, es posible que las expectativas fueran generadas 
por el contexto socioeconómico y cultural donde los estudios superiores son posibles y esperables.

6) Participación en el Programa Piloto de Tutores
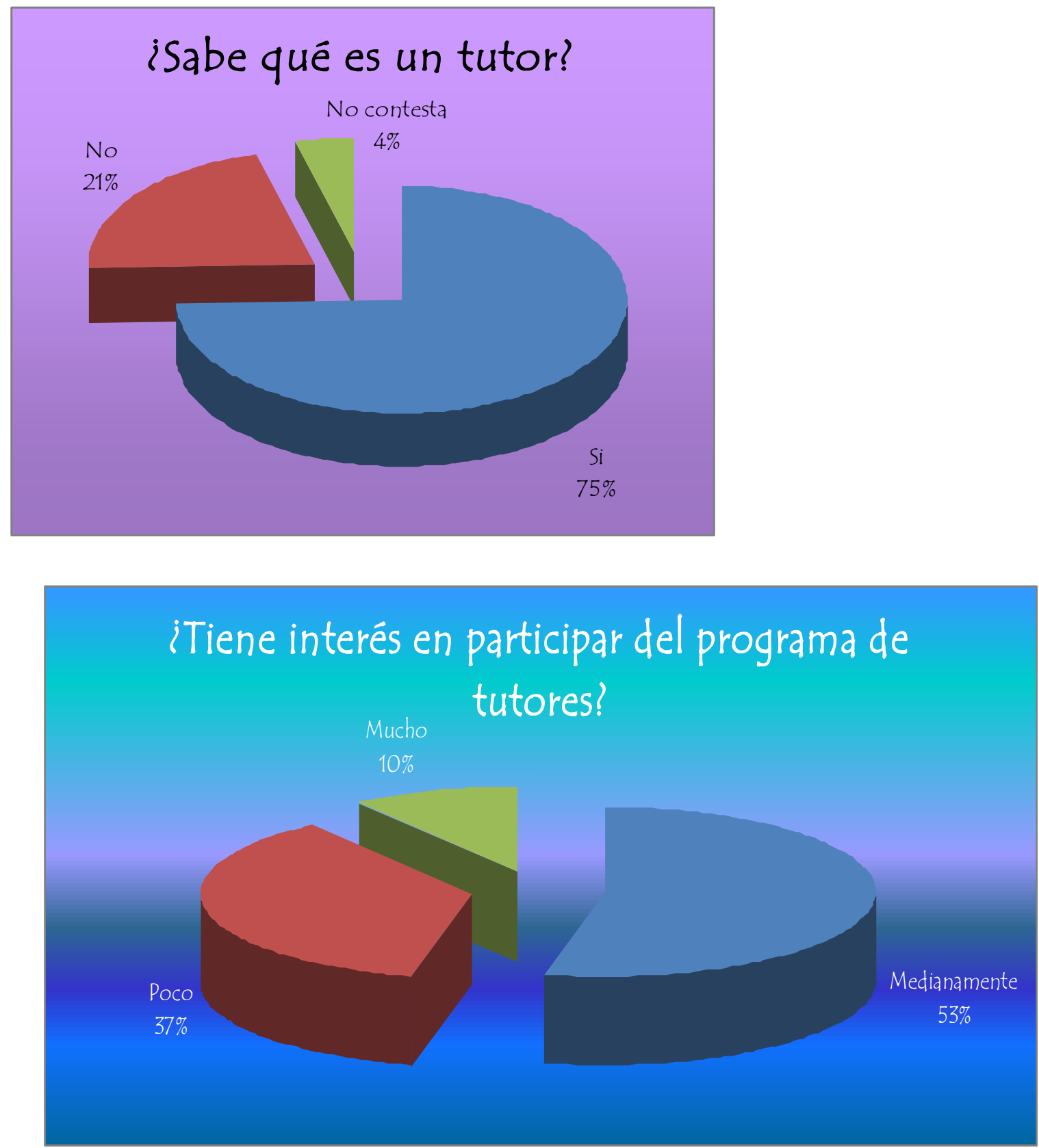

El cruce de la información expresada en los gráficos anteriores da como resultado la siguiente tabla. 


\begin{tabular}{|l|c|c|c|c|}
\hline \multirow{2}{*}{} & \multicolumn{3}{|c|}{ Interés en participar del programa de tutores } \\
\cline { 2 - 5 } & Poco & Medianamente & Mucho \\
\cline { 2 - 5 } & No contesta & 0 & 3 & 2 \\
\hline \multirow{3}{*}{ Sabe que es un tutor? } & Si & 104 & 166 & 27 \\
\cline { 2 - 5 } & No & 32 & 30 & 8 \\
\hline
\end{tabular}

A pesar de los resultados alentadores respecto a la figura del tutor y la incorporación al Programa de los ingresantes, obtenidos en la encuesta inicial. La asistencia a los horarios de consulta de tutores dispuestos especialmente antes o después de las cursadas de primer año han sido muy pobres. Cabe preguntarse si lo que los alumnos perciben como dificultades en los tramos iniciales, asociadas a la capacidad intelectual y al buen uso de las herramientas del aprendizaje es leído por ellos mismos, como dificultades en la introducción a la vida universitaria, por lo cual sería aconsejable la orientación de un tutor. También es posible que el Programa no haya sido legitimado aún, estableciendo resultados positivos palpables desde un grupo de alumnos significativo que sea observable por el resto de sus compañeros.

La tarea del programa actualmente, se enfoca en adquirir visibilidad y en construir la segunda encuesta a realizarse en el segundo cuatrimestre. Donde será posible identificar los problemas surgidos a los alumnos en el primer cuatrimestre de la cursada. Otro de los objetivos es sumar a los docentes, en especial de primer año, para que informen a los alumnos desde las mismas cátedras de su existencia y como verdadero complemento de sus estudios.

Esta experiencia se enmarca en una investigación-acción donde se espera que los jóvenes sean protagonistas de su proceso formativo-educativo y la institución brinde las herramientas a su alcance para la realización de las metas individuales, teniendo como horizonte el valor social de la formación universitaria.

El presente Programa puede poner de manifiesto algunas condiciones institucionales que pueden favorecer la inserción del joven en la universidad. Para apuntalarla tiene que estar acompañada de otras condiciones que van a sostener la posibilidad de que los alumnos realicen diferentes experiencias: un tiempo más extendido que les permita encontrar un ritmo adecuado, profesores que estén sintonizados con la experiencia, un seguimiento distante pero atento que posibilite una observación de comportamientos y actitudes. 


\section{GESTIÓN ACADÉMICA Y ESTRATEGIAS PARA LA RETENCIÓN DE LA MATRÍCULA: PROGRAMAS DE TUTORÍAS EN LA UNIVERSIDAD PÚBLICA}

Y sobre todo, demanda que pensemos a "los jóvenes no como víctimas pasivas de sus circunstancias sino como sujetos activos de su experiencia",7

Actualmente se siguen acumulando pruebas de que el abandono tiene que ver en gran medida también con los bajos niveles de estímulos intelectuales y sociales generados en interacciones personales con profesores, tutores $\mathrm{u}$ otros alumnos que parecen constituir un componente fundamental del proceso por medio del cual los sujetos son capaces de alcanzar sus metas educativas. ${ }^{8}$

\section{A modo de cierre...}

Estudiar una carrera universitaria a tiempo completo, es una opción y una oportunidad de vida que disocia al estudiante por un periodo de tiempo del mundo del trabajo. La educación adquiere una función preparadora o bien una función distribuidora de status, ya que la misma determina el acceso a determinados bienes y servicios, como así también, ubicaciones posibles en el sistema de empleo. Adentrándonos en el siglo XX, la relación entre educación y movilidad social se ha ido incrementando, ya que la educación permite o impide el acceso a ocupaciones de privilegio, y por ende a remuneraciones y status socioeconómico. Sin embargo hay que tener en cuenta el origen socioeconómico de los estudiantes a la hora de la efectivización del acceso al circuito de bienes y servicios, ya que la selección mayor se produce en los niveles educativos previos al superior. Aún si se creyera que la educación estuviera guiada por los requerimientos y los tiempos del sistema de empleo hay que asumir que la misma apunta a funciones más amplias y complejas como institución. ${ }^{9}$

De manera creciente la educación se volvió una determinante importante del empleo y la carrera. En general, los periodos de aprendizaje vocacional largos son premiados con un status más alto y con trabajos más interesantes e independientes. Sin embargo esto está afectado por las situaciones de oferta y demanda del mercado laboral. ${ }^{10}$

\footnotetext{
${ }^{7}$ Rodríguez, Daniel (2002; 195), citado por Emiliano Galende en "Subjetividad y Resiliencia: del azar a la complejidad", Miño Dávila.

${ }^{8}$ Tino Vincent (1993), “Leaving Collage, Rethinking the Cause and Cures of Student Attrition”, The University of Chicago Press, Chicago.

${ }^{9}$ Estas situaciones referidas por la bibliografía consultada a veces se contrasta en países que, como el nuestro, las crisis socio económicas son ya estructurales más que coyunturales. Esta situación hace que en momentos como el que vivimos se priorice la necesidad de técnicos y obreros calificados.

${ }^{10}$ Teichler, Ulrich. Graduados y empleo. Investigación metodológica y resultados. Los casos de Europa, Japón, Argentina y Uruguay.(2005:35)
}

Rev. GUAL., Florianópolis, v.2, n. 1, p.16-34, ano 2009. 


\section{GESTIÓN ACADÉMICA Y ESTRATEGIAS PARA LA RETENCIÓN DE LA MATRÍCULA: PROGRAMAS DE TUTORÍAS EN LA UNIVERSIDAD PÚBLICA}

Los desajustes entre oferta de profesionales universitarios, expansión de las matrículas universitarias y egresos y la debilidad de las estructuras ocupacionales; la extensión del llamado "efecto fila", la competencia por el empleo y no por el salario, los procesos de subcalificación, la vulnerabilidad y precariedad de la mayor parte de los puestos de trabajo han dado por tierra con las promesas que el proceso de reestructuración económica e integración económica a los mercados mundiales prometió en el marco de las reformas impuestas en los años noventa.

A través de los aportes de este breve trabajo se espera sumar a la resolución de la deserción de la población estudiantil, ya que se ha convertido en uno de los problemas más importantes de los últimos años en Educación Superior. Asimismo se pretende aportar a la dinámica de la Gestión Académica en estos nuevos escenarios que toca enfrentar.

\section{BIBLIOGRAFÍA}

ALVAREZ PEREZ. P. 2002. La función tutorial en la Universidad. Una Apuesta por la mejora de la calidad de la Enseñanza. ED. EOS, Madrid.

ALTBACH, P, McGrill Peterson, P, (editores), 2000, Educación Superior en el siglo XXI, Desafío Global y Respuesta Nacional, ED. Biblos, Buenos Aires.

ANTONI, J. 2003.Alumnos Universitarios, el por qué de sus éxitos y fracasos. Investigación que fundamenta una tesis doctoral. ED. Miño y Dávila. Buenos Aires.

ARANA, MARTA, 2006. El Programa de Incentivos bajo la lupa. El Caso de la UNMDP. Estanislao Balder, Mar del Plata. Argentina.

BOU MARÍA LUISA, ARANA MARTA, MENÉNDEZ SILVINA, Y OTROS “Gestión y retención de matricula universitaria: alumnos en tutorías en la FRA.UTN". Presentado en Coloquio Internacional en Gestión Universitaria en Amèrica del sSur. Mar del Plata. 2005.

ARANA MARTA, BOU MARIA LUISA "La retención de matricula en universidades públicas argentinas: el caso de la FRA-UTN" Presentado en Coloquio Internacional en Gestión Universitaria en Amèrica del Sur. Florianópolis. Brasil. 2004.

BOU, M.L., MENENDEZ S. Y GARAVENTA L "La implementación de un sistema de retención institucional en la FRA-UTN" Jornadas UDI, Rosario, 2004. Argentina

GARCIA de FANELLI, A,2002. Indicadores y estrategias en relación con el abandono Universitario. la Agenda Universitario.

Rev. GUAL., Florianópolis, v.2, n. 1, p.16-34, ano 2009. 


\section{GESTIÓN ACADÉMICA Y ESTRATEGIAS PARA LA RETENCIÓN DE LA MATRÍCULA: PROGRAMAS DE TUTORÍAS EN LA UNIVERSIDAD PÚBLICA}

GÓMEZ, M. 2001. El mercado de trabajo para los egresados universitarios recientes. En Aníbal Jozami / Eduardo Sánchez Martínez (comp) Estudiantes y profesionales en la Argentina. UNTref. Bs. As.

HOBSBAWN, Eric, 1995, Historia del Siglo XX, ED. Critica, Barcelona

KROSTCH, Pedro ( org.), Pratti, Marcelo ( editor), 2002, La Universidad Cautiva, Legados, Marcos y horizontes, ED, Ediciones al Margen, Bs. As.

KROSTCH, Pedro (comp.), 2003, Las Miradas de la Universidad, ED. Ediciones al Margen, Buenos Aires.

MICHAVILA, F, DELGADO. J,2005. La Tutoria y los nuevos modos de aprendizaje en la Universidad, Cátedra de La UNESCO de Gestión y Política Universitaria. ED. Comunidad de Madrid, Consejería de Educación. Dirección General de Universidades. Madrid.

MANSUY Michèle, THIREAU Véronique, JOSEPH Olivier (colab.), MARTINELLI Daniel (colab.) 2003. ¿Qué sectores para los principiantes?

MOLLIS, MARCELA, 2001, La Universidad argentina en Tránsito. Ensayo para jóvenes y no tan jóvenes. Fondo de Cultura Económica, Buenos Aires.

PANAIA, M. 2001. Trayectorias profesionales y demandas empresariales de ingenieros en la Argentina. Revista Latinoamericana de Estudios del Trabajo. Número especial dedicado a las Trayectorias ocupacionales y mercado de trabajo. Año 7.N 13

PANAIA, M. 2003 Trayectorias de ingenieros argentinos de perfiles tecnológicos En Revista de la Educación Superior Vol XXXII (1) Nº 125.

PANAIA, M. 2006.Trayectorias de Ingenieros Tecnológicos. Graduados y alumnos en el mercado de trabajo. Ed. Miño y Dávila. Buenos Aires.

PARRIÑO, 2005. De la Reflexión a la acción. Políticas para disminuir los procesos de deserción universitaria. En Efrón, M, Vega, R, (comp.) Aportes al debate sobre la gestión Universitaria II. Ed. De los cuatro Vientos. Bs As.

RIQUELME, G. 2004. Educación superior, demandas sociales, productivas y mercado. Miño y Dávila. Bs. As.

RODRIGUEZ ESPINAR. S, 2004 Manual de tutoría universitaria. Recursos para la acción. Ed. Octaedro, Barcelona.

SCHVARTZMAN, AMËRICO, STANG, MA FERNANDA. 1998. La Reforma Universitaria. La revolución de las conciencias. 1918-1998.Eduner. Entre Rios.

SUASNÁBAR, C, RINESI, E, SOPRANO, G (comp.), 2005. Universidad: Reformas y Desafíos, Prometeo Libros, Buenos Aires. 
TEICHLER, U, 2005. Graduados y empleo: Investigación, metodología y resultados. Los casos de Europa, Japón, Argentina y Uruguay. Ed. Miño y Dávila. Buenos Aires. 